\title{
Development of Portable Electrical-cell-substrate Impedance Sensing System
}

\author{
Chan-Young Park, ${ }^{1,2}$ Jae-Hong Min, ${ }^{1,2}$ Yu-Seop Kim, ${ }^{1,2}$ \\ Hye-Jeong Song, ${ }^{1,2}$ and Jong-Dae Kim ${ }^{1,2^{*}}$ \\ ${ }^{1}$ Department of Convergence, Hallym University, \\ 1 Hallymdaehak-gil, Chuncheon-si, Gangwon-do 24252, Korea \\ ${ }^{2}$ Bio-IT Research Center, Hallym University, \\ 1 Hallymdaehak-gil, Chuncheon-si, Gangwon-do 24252, Korea
}

(Received May 29, 2018; accepted September 26, 2018)

Keywords: ECIS, Wi-Fi, portable system, battery

We propose a compact electrical-cell-substrate impedance sensing (ECIS) system. The ECIS system measures the amount of microorganisms cultivated in an incubator and monitors it in real time. This is based on the measurement of the AC impedance change of a small goldfilm electrode on a culture well. In the existing system, except for sensors for impedance measurement, we needed external equipment such as a lock-in amplifier and a personal computer. Also, the sensors inside the incubator and the systems on the outside were connected by a wire. These make the laboratory complex and the system difficult to install. In this study, we implemented a small integrated ECIS system with a recent USB oscilloscope, a battery, and Wi-Fi technology to solve the above problems. System integration allows the entire system to be placed in an incubator, eliminating the need for external systems. Also, it is easy to carry and install it because of its simple structure. A Wi-Fi antenna was used to provide network connectivity inside the incubator to provide a web interface for status monitoring. The system is in the power-saving mode except when measuring to save on battery power. Experimental results on the relationship between measurement interval and battery capacity are given.

\section{Introduction}

Electrical-cell-substrate impedance sensing (ECIS) is a technique for monitoring the proliferation of living cells. It measures the impedance change by applying an alternating current of several $\mu \mathrm{A}$ or less through the small gold electrode of the culture dish. A lock-in amplifier is usually used to measure the impedance, where the receiving signal is analyzed with respect to the transmitting alternating current. ${ }^{(1)}$ The frequency of the current passing through the cell is normally within 1 to $100 \mathrm{kHz}$ and depends on the environment such as the experimental conditions and cell type. The measured transmitting and receiving signals can be analyzed by the digital lock-in or sine-fitting method. ${ }^{(2,3)}$ The morphology of animal cells responds not only to chemical, biological, or physical stimuli but also to changes in metabolism,

*Corresponding author: e-mail: kimjd@hallym.ac.kr https://doi.org/10.18494/SAM.2019.2091 
but does not directly affect the cells because the transmitting signal is less than several $\mu \mathrm{A}$. If the cell proliferates and changes its morphology, the current passing through will change and the measured impedance carries information on how much the cell has grown. ${ }^{(4)}$ In this regard, ECIS technology is used in a variety of experiments in the field of cell biology.

Studies on the spread of cancer cells using ECIS technology and the stimulation of drug development using stem-cell-based disease models have been actively carried out, such as studies on regenerative medicine or cell interaction and toxicity measurement. ${ }^{(5-8)}$ In addition, ECIS technology has recently demonstrated its potential as a rapid and reliable evaluator of the toxicity of drinking water. ${ }^{(9,10)}$

In a conventional ECIS system, various devices such as a lock-in amplifier, a PC, a well array station, and an incubator are required, and since it is usually used in a laboratory, it is very bulky and requires a constant power source. In particular, the size and power consumption of the lock-in amplifier dominate the entire system. Nowadays, a new compact USB oscilloscope can be used as an alternative to lock-in amplifiers. According to the USB specifications, the oscilloscope operates within 500 to $900 \mathrm{~mA}^{(11)}$ Also, it is small because there is no additional interface, such as the user interface, other than the measurement function. It is advantageous that less power is consumed in the system, given the difficulty of connecting a power supply in an incubator or in the field. ${ }^{(12,13)}$ Also, space is limited and the device should be small. In the long run, however, the power consumption of the USB oscilloscope is not very low. In this paper, we replace the lock-in amplifier, which is relatively large, with the latest compact USB oscilloscope with function generators.

Although the required power of the USB oscilloscope is relatively small, there is room for reducing its power consumption. Therefore, we also investigate ways to reduce its power. Because of the nature of cell proliferation, the conventional system is usually idle except during measurement. Therefore, leaving the system running during this idle time is a waste of power. To solve this problem, the system was completely shut down except during measurements, and the power required was reduced as much as possible. Experiments were performed with a $20000 \mathrm{mAh}$ battery to investigate how long it takes for an unoptimized system and an optimized system to consume the battery power. As a result, when the impedance was measured every $5 \mathrm{~min}$, the proposed optimized system operated for two days. If the measurement period was longer than $16 \mathrm{~min}$, the operation for more than one week could be expected. However, the unoptimized system was difficult to operate for more than one day regardless of the measurement period.

\section{Materials and Methods}

\subsection{System architecture}

A USB oscilloscope (Analog Discovery 2, Digilent, USA) was used for impedance measurement (Fig. 1). To measure cell proliferation, the signal frequency of the oscilloscope should be from 1 to $100 \mathrm{kHz}$. To measure the impedance, the voltage sampling rate should be $100 \mathrm{M}$ samples per second. A higher sampling rate did not significantly affect the error of the 


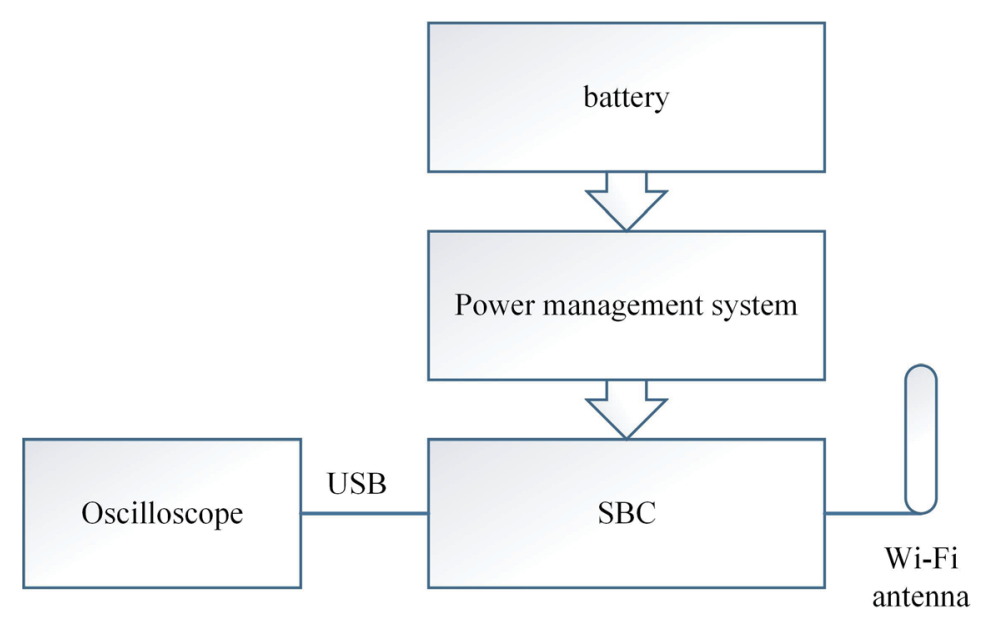

Fig. 1. (Color online) System architecture.

measurement results. The USB oscilloscope could not be operated by itself and it requires a standard computing device. Therefore, we employed a single-board computer (SBC) (Banana Pi M2U, Sinovoip Co., Ltd., China) for system operation. The SBC controlled the oscilloscope via a USB interface. In addition, a separate module was installed to measure the power consumption of the SBC system. This module was powered by a separate battery from the SBC and the power consumption was very small, so it did not affect the experiment results. A power management system was employed to supply or cut power to the SBC. The system supplied power to the SBC at a specified time to measure the impedance and the SBC did not consume power during the standby time. This minimized the overall energy consumption of the system. The scheduling should be registered in the SBC first and the user should select the measurement period considering the time from the activation of the system to the measurement. For the system used in this study, the measurement time was $2 \mathrm{~min}$, so the measurement cycle should be more than $3 \mathrm{~min}$ in order to benefit from energy saving through power management. At this time, in order to measure the power consumed by the system, a current shunt power monitoring chip module (INA219 module, Adafruit Industries, USA) was installed between the battery and the power connector of the power management system so that the amount of energy used could be measured. The module was connected to the PC and received power separately from the battery. The module also enabled the measurement of the power consumed even when the SBC was shut down.

The proposed ECIS system is shown in Fig. 2(b). In the figure, the emulator is connected to the oscilloscope instead of the culture well. The emulator was designed to mimic eight cell culture wells. It emulated different cell growth situations in 8 wells by connecting each of the resistances from 1 to $8 \mathrm{k} \Omega$ in parallel with $10 \mathrm{nF}$ capacitors. The system was located inside the incubator, as shown in Fig. 2(a), leaving only the antenna outside. The power was supplied from a $20000 \mathrm{mAh}$ battery. The normal voltage of the lithium ion battery was 3.6 to $4.2 \mathrm{~V}$. However, since the voltage used by the SBC was $5 \mathrm{~V}$, there was current loss during the step-up. Because the actual capacity was determined by the conversion efficiency of the battery, the amount of 


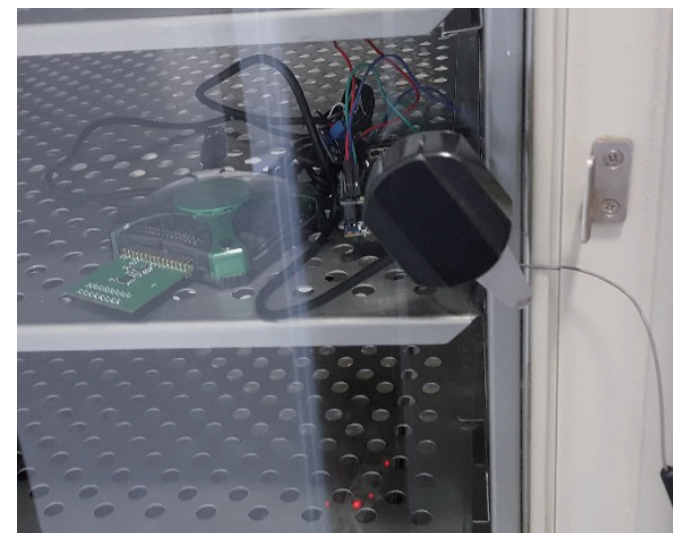

(a)

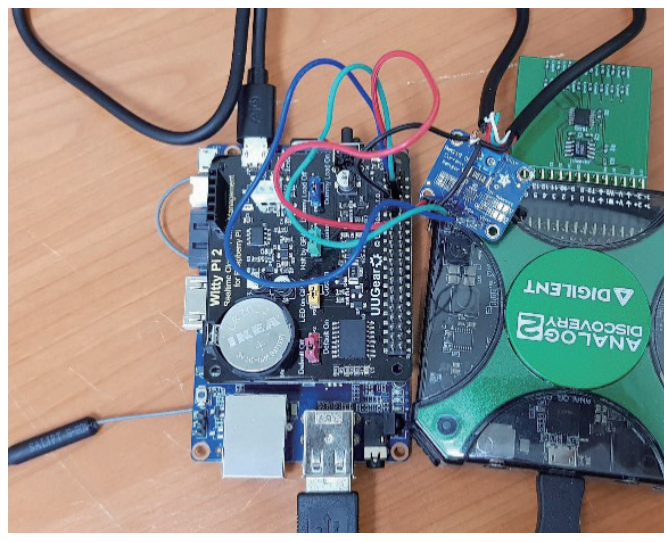

(b)

Fig. 2. (Color online) ECIS system viewed (a) from the top and (b) in the incubator.

energy available from the same $20000 \mathrm{mAh}$ battery might vary. For the batteries used in the experiment, the energy efficiency was $93 \%$ and the actual capacity was about $13000 \mathrm{mAh}$.

The power consumed in the above environment was measured. However, it was not measured in the system-off state because no power was consumed.

\subsection{Software architecture}

In Fig. 3, the SBC included the 'Current Checker', 'Client', and 'Scheduler' software modules. The Current Checker recorded the power consumed by the system. It measured current (mA), voltage (V), and power (W) every second, and stored the data locally. Measurement was started when the system booted up, but it was not needed in the power saving mode when power was not consumed. The Scheduler synchronizes the time of the SBC with the real time, and reserves the next boot time considering the measurement time and period. To accomplish accurate cycle-by-cycle measurement, the reservation was made at the system boot time earlier than the measurement time. In other words, since the measurement was prepared in advance before the actual measurement, it could be accurate. The Client module monitored the control parameters, such as system behavior, measurement cycle, and their changes, and they were input to the cloud database by the user. When the Scheduler detected the start command, the measurement of the impedance through the oscilloscope was begun. The measured data were also recorded in the database. Therefore, even if the SBC was in a power saving mode to save energy, the user could check the system status or the measured data. In addition, even if the system was booted sooner than expected, the time and measurement cycle were recorded at the same time as the experiment was started, ensuring accurate measurement time.

\subsection{Power consumption by system status}

Because there was a large difference in the power consumed by the system state, the states were classified as below. 


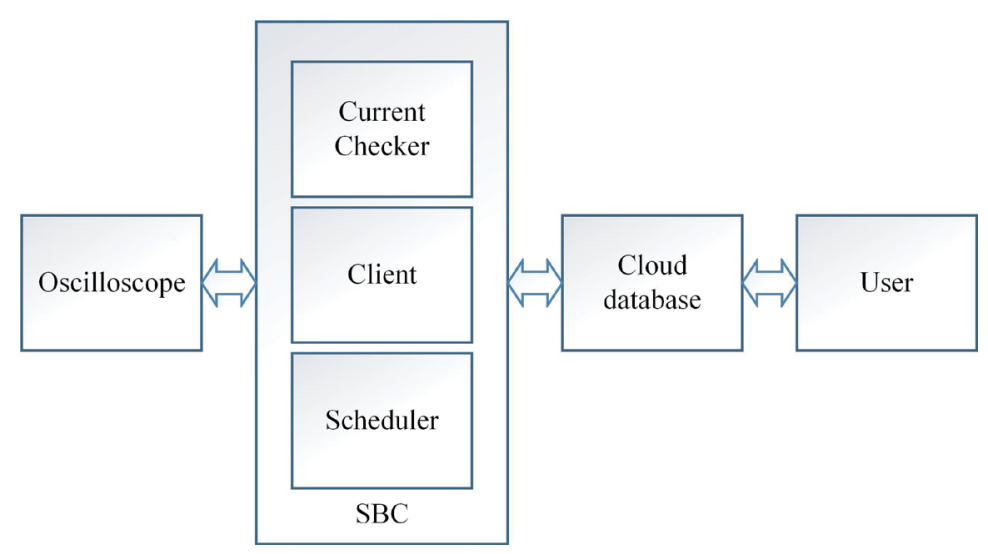

Fig. 3. (Color online) Software architecture.

1. Off: system off

2. Idle: system on, idle

3. Busy: system on, impedance measuring

If system operation was not absolutely necessary, the system was switched to a low-power state to conserve battery power. In the Off state, only the power management system was activated and minimum power was consumed. For impedance measurements, the power management system powered the SBC and the SBC was booted. This state was the Idle state in which the oscilloscope did not operate and only the SBC consumed energy. When booting was completed and the impedance measurement state was reached, the oscilloscope started operating and consumed much power (Busy state). When the measurement was completed, it went into the power saving mode again. Therefore, the longer the measurement period, the lower the average power consumption. However, if the measurement period was too long, the measurement results for cell proliferation became imprecise. Therefore, the system had an optimal period of measurement. In the system used in this study, the measurement time was 2 min. Therefore, in order to benefit from energy conservation through power management, the measurement period must be at least $3 \mathrm{~min}$. In this experiment, the impedance was measured every $5 \mathrm{~min}$. Therefore, the power-saving mode was set for the remaining $3 \mathrm{~min}$. Considering that the cell culture doubling time was usually one day, the interval of 5 min was not long. ${ }^{(14)}$

\section{Results}

In the Off state, the power consumption was negligible and not measured. We measured the power consumption in the Idle state for about $11 \mathrm{~h}$ and the Busy state for about $21 \mathrm{~h}$. Figures 4 and 5 show the changes in the current consumption in the Idle and Busy states, respectively.

A current in the range of 300-400 mA was consumed in the Idle state, as shown in Fig. 4. It was sometimes measured as being higher, but its average value of $326.34 \mathrm{~mA}$ did not significantly change. 


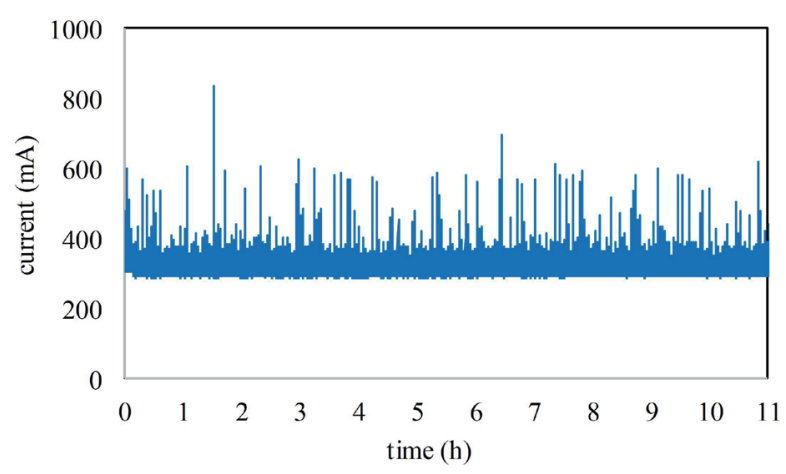

Fig. 4. (Color online) Current consumption change when the system was in the Idle state.

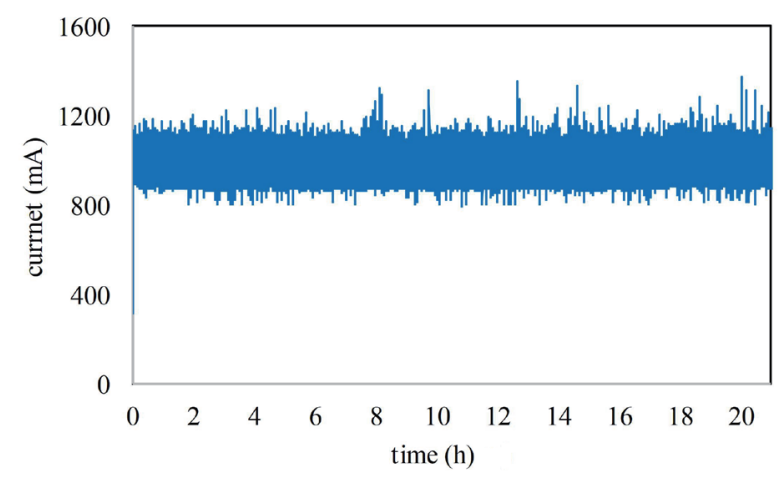

Fig. 5. (Color online) Current consumption change when the system was in the Busy state.

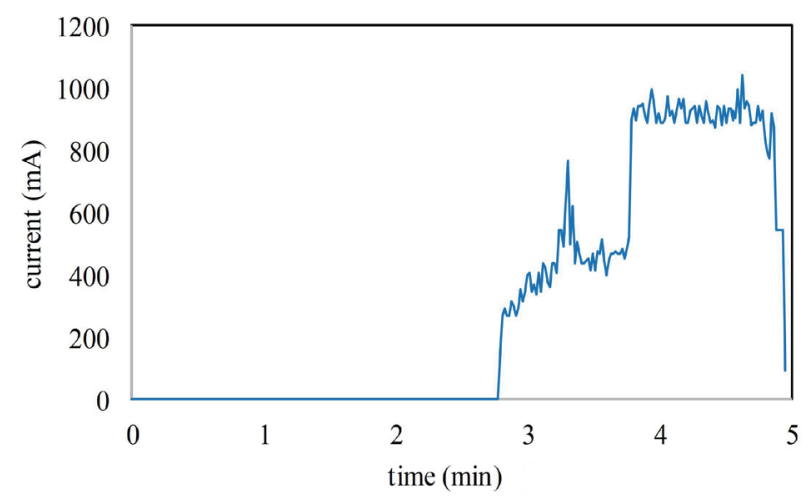

Fig. 6. (Color online) Energy consumption in one measurement cycle with power management.

On the other hand, in the Busy state, it consumed 800-1200 mA current, as shown in Fig. 5, which was about three times higher than that in the Idle state. Since an average of $954.1 \mathrm{~mA}$ of current was consumed, a large amount of battery energy would be consumed if the Busy state was always maintained.

Figure 6 shows the change in the consumed current when power management was applied and the measurement period was $5 \mathrm{~min}$. With power management, the average power consumed in each measurement cycle was $293 \mathrm{~mA}$. In this case, the system consumed half the average current of $625.3 \mathrm{~mA}$ required when the system was kept idle instead of sleeping. The proposed system with power management could be operated for two days with a $20000 \mathrm{mAh}$ battery, whereas the existing system without power management was operated for one day. As the power-saving time becomes longer, the operation time lengthens. As a result, the system operation time varies depending on the ratio of the power saving time to the measurement time. 


\section{Conclusions}

In this paper, we proposed a portable ECIS system and investigated a method of minimizing battery power consumption. The proposed compact system was composed of the latest oscilloscope, SBC, and Wi-Fi technology, and the resultant system could be fitted into an incubator. A power management method was also proposed to save battery power. With the proposed method, the longer the power-saving state than the measurement time of $2 \mathrm{~min}$, the longer the proposed system could be operated. If there was no power-saving state, the system could operate only for 1 day. However, the proposed method enabled operation for about 2 days. In order to operate the system for more than one week, the proposed system requires a measurement interval of at least $16 \mathrm{~min}$. Once the application is determined, the proposed methodology can be used to determine system requirements such as measurement period and battery capacity.

\section{Acknowledgments}

This research was supported by the Basic Science Research Program through the National Research Foundation of Korea (NRF) funded by the Ministry of Science, ICT \& Future Planning (NRF-2017R1A2B4002679) and The Leading Human Resource Training Program of Regional Neo Industry through the National Research Foundation of Korea (NRF) funded by the Ministry of Science, ICT \& Future Planning (NRF-2016H1D5A1909654).

\section{References}

1 I. Giaever and C. R. Keese: PNAS 88 (1991) 7896.

2 M. Rosu-Hamzescu, S. Oprea, C. Polonschii, E. Gheorghiu, and M. Gheorghiu: 21st Int. Conf. Control Systems and Computer Science (CSCS, 2017) 69.

3 P. M. Ramos and A. C. Serra: IEEE Trans. Instrum. Meas. 57 (2008) 197.

4 N. Hadjout, G. Laevsky, D. A. Knecht, and M. A. Lynes: Biotechniques 31 (2001) 1130.

5 P. O. Bagnaninchi and N. Drummond: PNAS 108 (2011) 6462.

6 T. B. Tran, C. Baek, and J. Min: PLOS ONE 11 (2016) e0153813.

7 D. Mondal, D. Pal, and C. Roy Chaudhuri: J. Appl. Phys. 118 (2015) 044701.

8 Y. Xu, Y. Lv, L. Wang, W. Xing, and J. Cheng: Biosens. Bioelectron. 32 (2012) 300.

9 M. W. Widder, L. M. Brennan, E. A. Hanft, M. E. Schrock, R. R. James, and W. H. Schalie: J. Appl. Toxicol. 35 (2015) 701.

10 L. M. Brennan, M. W. Widder, L. E. Lee, and W. H. van der Schalie: Toxicol. in Vitro 26 (2012) 736.

11 F. He: IEEE Symp. Product Compliance Engineering (ISPCE) (IEEE, 2015$) 1$.

12 C. Liu, C. E. Banister, C. C. Weige, D. Altomare, J. H. Richardson, C. M. Contreras, and P. J. Buckhaults: Proc. National Academy of Sciences (2018) 201802902.

13 N. Pavillon, A. J. Hobro, S. Akira, and N. I. Smith: PNAS (2018) 201711872.

14 F. Witzel, R. Fritsche-Guenther, N. Lehmann, A. Sieber, and N. Blüthgen: Bioinformatics 31 (2015) 2705. 